\title{
Glass transition dynamics of anti-inflammatory ketoprofen studied by Raman scattering and terahertz time-domain spectroscopy
}

Tomohiko Shibata*, Haruki Takayama, Tae Hyun Kim, Tatsuya Mori, and Seiji Kojima

Graduate School of Pure and Applied Sciences, University of Tsukuba, Tsukuba, Ibaraki 305-8573, Japan

\begin{abstract}
A liquid-glass transition and a crystalline state of pharmaceutical racemic ketoprofen were studied by Raman scattering and the broadband terahertz time-domain spectroscopy (THz-TDS) in the frequency range from 9 to $260 \mathrm{~cm}^{-1}$. The low-frequency Raman scattering spectra clearly shows the remarkable change related to a liquid-glass transition at about $T_{\mathrm{g}}=267$ $\mathrm{K}$. After melt-quenching at liquid nitrogen temperature, a boson peak appears at about $16.5 \mathrm{~cm}^{-1}$ near and below $T_{\mathrm{g}}$ and the intensity of quasi-elastic scattering related to structural relaxation increases markedly on heating. The crystalline racemic ketoprofen of "conformer $A$ " shows the noncoincidence of mode frequencies below $200 \mathrm{~cm}^{-1}$ between Raman scattering spectrum and imaginary part of dielectric spectrum observed by THz-TDS.
\end{abstract}

Keywords: Ketoprofen; Boson peak; Raman scattering; THz-TDS; Glass transition; Crystal

*E-mail address: $\underline{\text { s1320438@u.tsukuba.ac.jp }}$

TEL: $+81-29-853-5262$ 


\section{Introduction}

In the pharmaceutical research, there is a growing interest currently in the development of glassy pharmaceuticals, because they often show a better solubility than crystalline pharmaceuticals $[1,2]$. However, one concern is the instability of a glassy state originating from the disordered structure and aging effect. Therefore, it is important to make clear the molecular dynamics in glassy states of pharmaceutical system for getting predictable stability. Up to now, vibrational spectroscopy such as FTIR and Raman scattering has been applied to study liquid-glass transitions of organic and inorganic glass forming materials [3-6]. In particular, the inelastic scattering spectra of glasses have generally shown a low-frequency response called "boson peak" and have never been satisfactorily understood. The investigation of the low-frequency Raman spectra of glassy pharmaceuticals will give new insights into the low-energy excitation of glassy materials and physical properties of glassy pharmaceuticals.

Terahertz time-domain spectroscopy (THz-TDS) and low-frequency Raman scattering spectroscopy have been successfully used for the identification and quantification of various solid state of drug, such as enantiomer, polymorphic, amorphous state [7-10]. It is because the spectral features in the low-frequency region below $100 \mathrm{~cm}^{-1}$ is associated with intermolecular vibration modes of molecules. For the characterization of pharmaceutical materials, it is important to measure both THz-TDS and low-frequency Raman scattering. The two methods have the complimentary selection rules which enable the full information on vibrational properties related to the crystalline structure, and their strength of intermolecular interactions.

Ketoprofen is one of nonsteroidal anti-inflammatory drugs (NSAIDs) used for the treatment of inflammation and pain. It was found by calorimetric measurements that the glassy ketoprofen can easily been achieved by rapid cooling from the liquid without crystallization [11-12]. Dynamical properties of glassy state and liquid-glass transition have been studied by dielectric spectroscopy in the frequency range from a few $\mathrm{Hz}$ to $\mathrm{MHz}$ [13]. Besides the primary $\alpha$-relaxation process above $T_{g}$, secondary $\gamma$-relaxation related to the localized motion has been found in glassy state. High frequency vibrational properties of a glassy and supercooled liquid ketoprofen are still unexplored. Vibrational properties of crystalline ketoprofen have been investigated by infrared, Raman scattering and inelastic neutron scattering spectroscopy in the frequency range of $100 \sim 3500 \mathrm{~cm}^{-1}$ [14-18]. In addition to the Raman and FTIR spectra of crystalline states, DFT calculations were reported by M.L. Vueba et al [18]. It has been reported that the bands assigned to $\mathrm{C}=\mathrm{O}$ and $\mathrm{O}-\mathrm{H}$ stretching vibrations display a downward shift relative calculated values for the isolated molecules due to intermolecular hydrogen bond type interactions. To get more information of these intermolecular interaction, low-frequency vibrational properties below $100 \mathrm{~cm}^{-1}$ is required. 
The present study is focused on the vibrational properties of both glassy and crystalline ketoprofen in a few THz range. For this, Raman scattering and THz-TDS were used. In this paper, we report the experimental results of the temperature dependence of low-frequency Raman scattering spectra in glassy, supercooled and liquid states of ketoprofen. The terahertz time-domain and Raman spectra of crystalline ketoprofen in the region of the low-frequency infrared vibrations have been measured and compared at room temperature.

\section{Experimental}

Ketoprofen ((RS)-2-(3-benzoylphenyl) propanoic acid, $\mathrm{C}_{16} \mathrm{H}_{14} \mathrm{O}_{3}$, a molar mass of 254.28 g/mol) was purchased from Sigma Co. (catalogue No. L1751 (CAS 22071-15-4), 98\% GC assay). It is a racemic mixture of S-(+)-ketoprofen and R-(-)-ketoprofen (see Figure 1) and the studied $( \pm)$-ketoprofen mixture is referred as ketoprofen. Ketoprofen is white crystalline powder at room temperature and used without further purification.

Raman scattering spectra were measured in the frequency range from 9 to $260 \mathrm{~cm}^{-1}$, in $V V$ and $V H$ geometry under a scattering angle $\theta=180^{\circ}$, using a single frequency green-YAG laser with wavelength $532 \mathrm{~nm}$. The spectrometer is a triple-grating monochrometer (T64000, Horiba-Jobin-Yvon) with additive dispersion [5] and the spectral resolution of the Raman spectrometer was $1.6 \mathrm{~cm}^{-1}$. A heating/cooling stage (Linkam, THMS600) was used to control the temperature of the sample from 90 to $373 \mathrm{~K}$. The temperature stability of a sample was within \pm $0.1 \mathrm{~K}$. In order to investigate the Raman scattering of crystalline, glassy, supercooled and liquid ketoprofen, the following two series of measurements were performed. First, crystalline ketoprofen was measured at room temperature, using a pressed pure pellet with $0.5-1.5 \mathrm{~mm}$ thickness. Crystalline sample was measured without analyzer. Second, the crystalline ketoprofen was heated and kept 5 minutes at $373 \mathrm{~K}\left(T_{m} \sim 368 \mathrm{~K}[12,13]\right)$ to get an equiilibrium liquid phase and then the liquid ketoprofen was quenched to liquid nitrogen temperature with high cooling rate of more than $10 \mathrm{~K} / \mathrm{min}$, which was high enough to obtained glassy ketoporfen. The Raman spectra of glassy, supercooled and liquid ketoprofen were recorded upon heating from $90 \mathrm{~K}$ to $373 \mathrm{~K}$.

$\mathrm{THz}$ transmission spectra were measured in the frequency range from 0.5 to $6.5 \mathrm{THz}(16.65$ to $216.45 \mathrm{~cm}^{-1}$ ) using a THz-TDS equipment (TAS7500SU, Advantest Co.) with a Cherenkov type broadband $\mathrm{THz}$ generator and the high-speed AOS technique [15]. Crystalline ketoprofen was measured only at room temperature, using the same sample of Raman measurement. 


\section{Results and discussion}

\section{3-1. Liquid-glass transition}

Ketoprofen undergoes a liquid-glass transition at $T_{\mathrm{g}}=267 \sim 270 \mathrm{~K}$ [11-13]. The temperature dependence of the low frequency Raman spectra was measured upon heating from the glassy state to equilibrium liquid state through a liquid-glass transition.

Boson peak is a common nature of the low-energy excitations of glassy materials and observed in inelastic scattering spectra such as Raman spectra of various glass formers, for example, lower alcohols and oxide glasses [5, 6]. As a general trend, a strong glass shows an intense boson peak and a weak fast relaxation process, while a fragile one shows a weak boson peak and an intense fast relaxation process [16]. The degree of fragility is given by the fragility index $m$ [10, 17] defined by

$m=\lim _{T \rightarrow T_{g}}\left|\frac{d \log \tau}{d\left(T_{g} / T\right)}\right|$,

where $\tau$ is the relaxation time of $\alpha$-relaxation.

Figure 2(a) shows the temperature dependence of $V H$ depolarized Raman scattering spectra of a glassy state of ketoprofen below $T_{\mathrm{g}}$. A boson peak appears at about $16.5 \mathrm{~cm}^{-1}$ and two intramolecular vibration modes appear at 75 and $220 \mathrm{~cm}^{-1}$. The boson peak spectrum of reduced intensity was fitted by log-normal function is shown in Figure 3. The reduced intensity $I_{r}(\omega)$ was defined by

$$
I_{r}(\omega)=\frac{I(\omega)}{\omega\{n(\omega)+1\}}
$$

where $I(\omega), n(\omega)$ is the Raman scattering intensity and the Bose-Einstein factor, respectively.

The intensity of a boson peak is weaker than that of glycerol, and the peak frequency is about a half of glycerol. Considering the fragility of glycerol is $m=50 \sim 54$ [18], weaker intensity is reasonable. Since the large value of fragility index of ketoprofen, $m=86$, was reported very recently [10], the boson peak intensity can be rather weak. The strong correlation is known between the boson peak frequency with the shear modulus [6], and the recent simulation predicted that the boson peak frequency is Ioffe-Regal limit of transverse acoustic mode [19]. Therefore, the lower boson peak frequency of ketoprofen may be caused by smaller shear modulus of glassy ketoprofen than that of glassy glycerol, which has a strong network of hydrogen bonds. 
Figure 2(b) shows the temperature dependence of Raman scattering spectra of supercooled and liquid states of ketoprofen above $T_{\mathrm{g}}$. With increasing temperature, a boson peak becomes weak and quasielastic scattering related to structural relaxation gradually grows and becomes intense. Supercooled liquid has never been recrystallized and behave like normal liquid during the measurement of Raman scattering. No recrystallization has been also observed in the calorimetric measurements [12]. It is a special feature of ketoprofen molecules in contrast to the fact that similar NSAIDs such as ibuprofen and aspirin exhibit strong tendency of recrystallization [20, 21]. Silicon dioxide $\mathrm{SiO}_{2}$ is polymorphic and more than ten kinds of crystalline states were reported, and silica glass has a strong glass-forming tendency. The existence of nine deferent geometries was reported by the density functional theory (DFT) calculations as the energy minimum conformations in crystalline states of ketoprofen [14]. The correlation between the glass formation and the number of polymorphic modifications was reported in chalcogenide and other glassy systems [22]. Therefore, the highly polymorphic nature of ketoprofen may prevent from forming a periodic alignment of molecules and show strong glass-forming tendency in contrast to other NSAIDs. This strong glass-forming tendency is suitable to study slow processes of a glass transition.

\section{3-2. Vibrational properties of crystalline state}

In THz-TDS, the inclusion of a sample causes the uncertainty to determine a dielectric constant of a sample. Therefore, any other solid powders such as polyethylene were not mixed into a powder sample to determine dielectric constant accurately. Figure 4 shows (a) the low-frequency $V O$ Raman scattering spectrum and (b) the imaginary part of a dielectric constant of crystalline ketoprofen at room temperature. Ketoprofen exhibits intense low-frequency peaks in Raman scattering and imaginary part of dielectric spectra originate from the intermolecular vibration modes in a crystalline state. We decomposed the measured spectra using multi-peak fitting procedure. Peaks are described by spectral functions of dumped harmonic oscillators (DHO) to determine the mode frequency and damping of each mode. Fitting lines are shown in Figure 4 and obtained peak frequencies were collected in Table 1 with the reported results of DFT calculation and vibrational assignment [17].

The comparison of fitted parameters between Raman scattering and THz-TDS spectra in the frequency range from 10 to $260 \mathrm{~cm}^{-1}$ was shown in Table 1 . In a Raman spectrum, the peaks above $100 \mathrm{~cm}^{-1}$ such as $128,156,170$ and $220 \mathrm{~cm}^{-1}$, are in agreement with the calculated values of the "conformer $A$ " reported in ref. 17 within experimental uncertainty. For the external modes below $200 \mathrm{~cm}^{-1}$, the difference of peak frequencies was clearly observed between and Raman scattering and THz-TDS spectra as shown in Table 1. In contrast, according to the previous study, the most of mode frequencies observed by Raman and FTIR measurements are in good 
agreement for the internal vibrational modes above $400 \mathrm{~cm}^{-1}$ [17]. It means that some vibrational modes are only Raman active, whereas others are only IR active in below $200 \mathrm{~cm}^{-1}$, while most of the vibrational modes are Raman and IR active above $400 \mathrm{~cm}^{-1}$.

Crystal structure of racemic ketoprofen has not been published up to now. However, it has been reported that racemic crystals tend to have a crystal structure which has a center of symmetry because of the existence of R- and S- enantiomers in it [26]. If a crystal has an inversion center, the mutual exclusion principle holds between Raman and IR activities. For crystalline ketoprofen, it seems that the exclusive principle holds for external vibration modes below $200 \mathrm{~cm}^{-1}$, while it may not hold for internal vibration modes above $400 \mathrm{~cm}^{-1}$. Our experimental results suggest that ketoprofen have a crystal structure which has a center of symmetry, while the orientations of molecule at lattice point dynamically disordered. This kind of orientationally disordered crystal has been reported in S- and racemic camphor at room temperature [27].

Since the low-frequency Raman and infrared spectra of ketoprofen have not been reported so far, direct comparison with previous results is not possible unfortunately. However, there are some information available on benzophenone $\left[\left(\mathrm{C}_{6} \mathrm{H}_{5}\right)_{2} \mathrm{CO}\right.$, containing two phenyl rings connected through a $\mathrm{C}=\mathrm{O}$ carbonyl group] which is similar to ketoprofen molecules. Ketoprofen molecules have carboxylic acid group which is related to hydrogen bonding, while benzophenone molecules do not have such functional group. Low-frequency vibrational modes of stable $\alpha$-form benzophenon have been observed in Raman measurement at 37, 63, 106 and $126 \mathrm{~cm}^{-1}[28,29]$. Considering the fact that the Raman modes of ketoprofen appear at 34, 57, 110 and $128 \mathrm{~cm}^{-1}$, it can be attributed to the same kind of vibrational mode reported in benzophenone $[28,29]$. On the other hands, some Raman modes at 46, 68 and $97 \mathrm{~cm}^{-1}$ which have been observed only in ketoprofen probably originate from the presence of carboxylic acid group. Some previous Raman spectroscopy and FT-IR studies suggested that ketoprofen molecules form cyclic hydrogen-bonded dimers in crystal structure, with pairs of $\mathrm{O}-\mathrm{H} \cdots \mathrm{O}$ hydrogen bonds between the carboxylic acid groups [14, 17]. Therefore, one possible origin of intense low-frequency peaks such as 46, 68 and $97 \mathrm{~cm}^{-1}$ in Raman scattering spectrum is the Raman active distortions of ketoprofen hydrogen bonded dimer that presence in the crystal structure.

\section{Conclusion}

Vibrational properties of pharmaceutical ketoprofen was studied in liquid, glass, and crystalline states by the low-frequency Raman scattering and the broadband THz-TDS in the 
frequency range between 9 and $260 \mathrm{~cm}^{-1}$. The low-frequency Raman scattering spectra clearly show the remarkable change related to a liquid-glass transition at about $T_{\mathrm{g}}=267 \mathrm{~K}$. A boson peak clearly appears at about $16.5 \mathrm{~cm}^{-1}$ at low temperatures below $T_{\mathrm{g}}$, and the intensity of quasi-elastic scattering related to structural relaxation increases markedly upon heating above $T_{\mathrm{g}}$. No recrystallization was observed in a supercooled liquid state, because the highly polymorphic nature of ketoprofen may prevent from forming a periodic alignment of molecules. The crystalline ketoprofen of "conformer $A$ " shows the difference of peak frequencies below $200 \mathrm{~cm}^{-1}$ between Raman scattering spectrum and imaginary part of dielectric spectrum of THz-TDS.

\section{Acknowledgement}

Authors are very thankful for the technical support on THz-TDS measurements to Sendai Office, Advantest Corporation. This work was partially supported by the Murata Science Foundation.

\section{References}

[1] B.C. Hancock, G.Zorgrafi, J. Pharm. Sci. 86 (1997) 1.

[2] L.Yu, Adv. Drug Deliver Rev. 48 (2001) 27.

[3] C. A. Angell, J. Wong, J. Chem. Phys. 53 (1970) 2053.

[4] N.J. Tao, G. Li, X. Chen, W.M. Du, H.Z. Cummins, Phys. Rev. A 44 (1991) 6665.

[5] S. Kojima, Phys. Rev. B 47(1993) 2924.

[6] S. Kojima, Y. Matsuda, Y. Fukawa, M. Kawashima, Y. Moriya, T. Yamada, O. Yamamuro, M. Kodama, J. Non-Cryst. Solids 356 (2010) 2524.

[7] M.D. King, W.D. Buchanan, T.M. Korter, J. Pharm. Sci. 100 (2011) 1116.

[8] M. Otsuka, J. Nishizawa, N.Fukura, T. Sasaki, J. Infrared Milli. Terahz Waves 33 (2012) 953.

[9] A. Hedoux et al., Eur. J. Pharm. Sci. 38 (2009) 156.

[10] C.M. McGoverin, T. Rades, K.C. Gordon, J. Pharm. Sci. 97 (2008) 4598.

[11] U. Sailaja et al., Eur. J. Pharm. Sci. 49 (2013) 333.

[12] J.A. Baird, B.V. Eerdenbrugh, L.S. Taylor, J. Pharm. Sci. 99 (2010) 3787.

[13] P. Di Martino et al., J. Cryst. Growth 265 (2004) 302.

[14] S.-H. Choi et al., Anal. Sci. 17 (2001) i785.

[15] L.A.E. Batista de Carvalho et al., Biopolymer 82 (2006) 420

[16] Y. H. Lu, C.B. Ching, Chirality 16 (2004) 16541

[17] M.L. Vueba, M.E. Pina, F. Veiga, J.J. Sousa, L.A.E Batista de Carvalho, Int. J. Pharm. 
307 (2006) 56.

[18] A. Bartels et al., Appl. Phys. Lett. 88(2006) 041117.

[19] A.P. Sokolov, E. Roessler, A. Kiskiukand, D. Quitman, Phys. Rev. Lett.. 71 (1993) 2062.

[20] R. Böhmer, K.L. Ngai, C.A. Angell, D.J. Plazek, J. Chem. Phys. 99 (1993) 4201.

[21] S. Pawlus, M. Paluch, J. Ziolo, C.M. Roland, J. Phys. Condens. Matter 21 (2009) 332101.

[22] H. Shintani, H.Tanaka, Nat. Mater. 7 (2008) 870.

[23] J.-H. Ko, K.-S. Lee, Y. Ike, S. Kojima, Chem. Phys. Lett. 465 (2008) 36.

[24] J.-H. Ko, T.H. Kim, K.-S. Lee, S. Kojima, Chem. Phys. Lett. 515 (2011) 221.

[25] L.N. Blinov, Glass Phys. Chem. 30 (2004) 180.

[26] C.P. Brock, W. B. Schweizer, J.D. Dunitz, J. Am. Chem. Soc., 113 (1991) 9811.

[27] I.B. Rietveld et al., Thermochimica Acta 511 (2010) 43

[28] L. Babkov et al, J. Mol. Struct., 792 (2006) 73.

[29] T.M. Kolev, B.A. Stamboliyska, Spectrochim. Acta A, 56 (1999) 119. 
Table 1. Comparison of mode frequencies $\left(\mathrm{cm}^{-1}\right)$ between Raman scattering and THz-TDS in crystalline ketoprofen of conformer $A$.

\begin{tabular}{|c|c|c|c|c|}
\hline \multirow{2}{*}{$\frac{\text { THz-TDS }}{\text { Observed }}$} & \multicolumn{2}{|c|}{ Raman } & \multirow{2}{*}{$\frac{\text { Calculated }^{[17]}}{\text { Conformer } A}$} & \multirow[t]{2}{*}{ Approximate description } \\
\hline & \multicolumn{2}{|c|}{ Observed $\quad$ Ref.14 } & & \\
\hline & 34 & & & \\
\hline \multirow[t]{3}{*}{41} & & & & \\
\hline & 46 & & & \\
\hline & 57 & & & \\
\hline \multirow{2}{*}{\multicolumn{5}{|c|}{$\begin{array}{l}79 \\
94\end{array}$}} \\
\hline & & & & \\
\hline & 97 & & & \\
\hline & 110 & 110 & & \\
\hline \multicolumn{5}{|l|}{114} \\
\hline & 128 & 124 & 117 & $C^{7}-\Phi-C^{8}$ wagging \\
\hline 139 & & 138 & 136 & $\mathrm{C}^{7}=\mathrm{O}^{13}$ out of plane bending \\
\hline \multirow{2}{*}{158} & 156 & 157 & 148 & $\Phi^{\prime}-C^{7}$ wagging \\
\hline & 170 & 170 & 184 & $\mathrm{CH}_{3}-\mathrm{C}-\Phi$ deformation; $\mathrm{C}^{7}-\Phi-\mathrm{C}^{8}$ wagging \\
\hline \multirow{2}{*}{\multicolumn{5}{|c|}{ 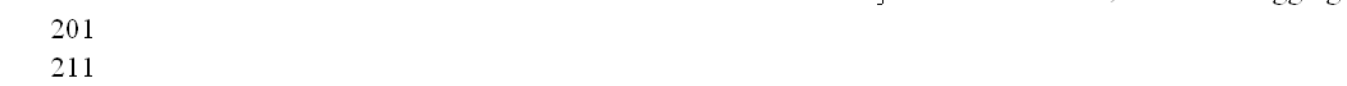 }} \\
\hline & & & & \\
\hline & 219 & 220 & 220 & $C^{7}-\Phi-C^{8}$ bending; $\Phi^{\prime}-C^{7}-\Phi$ deformation \\
\hline
\end{tabular}




\section{Figure captions}

Figure 1. Chemical structure of ketoprofen molecule, where $\mathrm{C}^{*}$ is a chiral carbon atom.

Figure 2. Temperature dependence of low-frequency Raman scattering spectra of (a) glassy ketoprofen and (b) supercooled liquid and liquid ketoprofen.

Figure 3. Reduced Raman scattering spectrum of ketoprofen around a boson peak at $193 \mathrm{~K}$.

Figure 4. (a) Raman scattering spectrum and (b) imaginary part of dielectric constant determined by THz-TDS in crystalline ketoprofen of conformer $A$. 
Figure 1

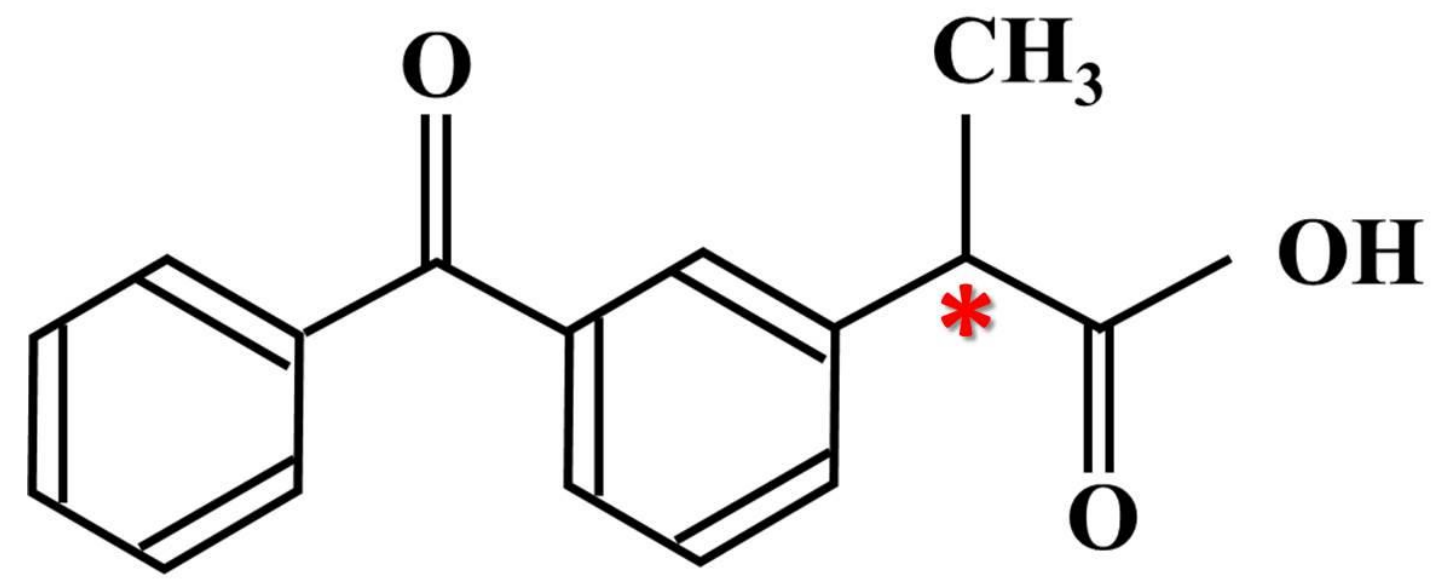


Figure 2

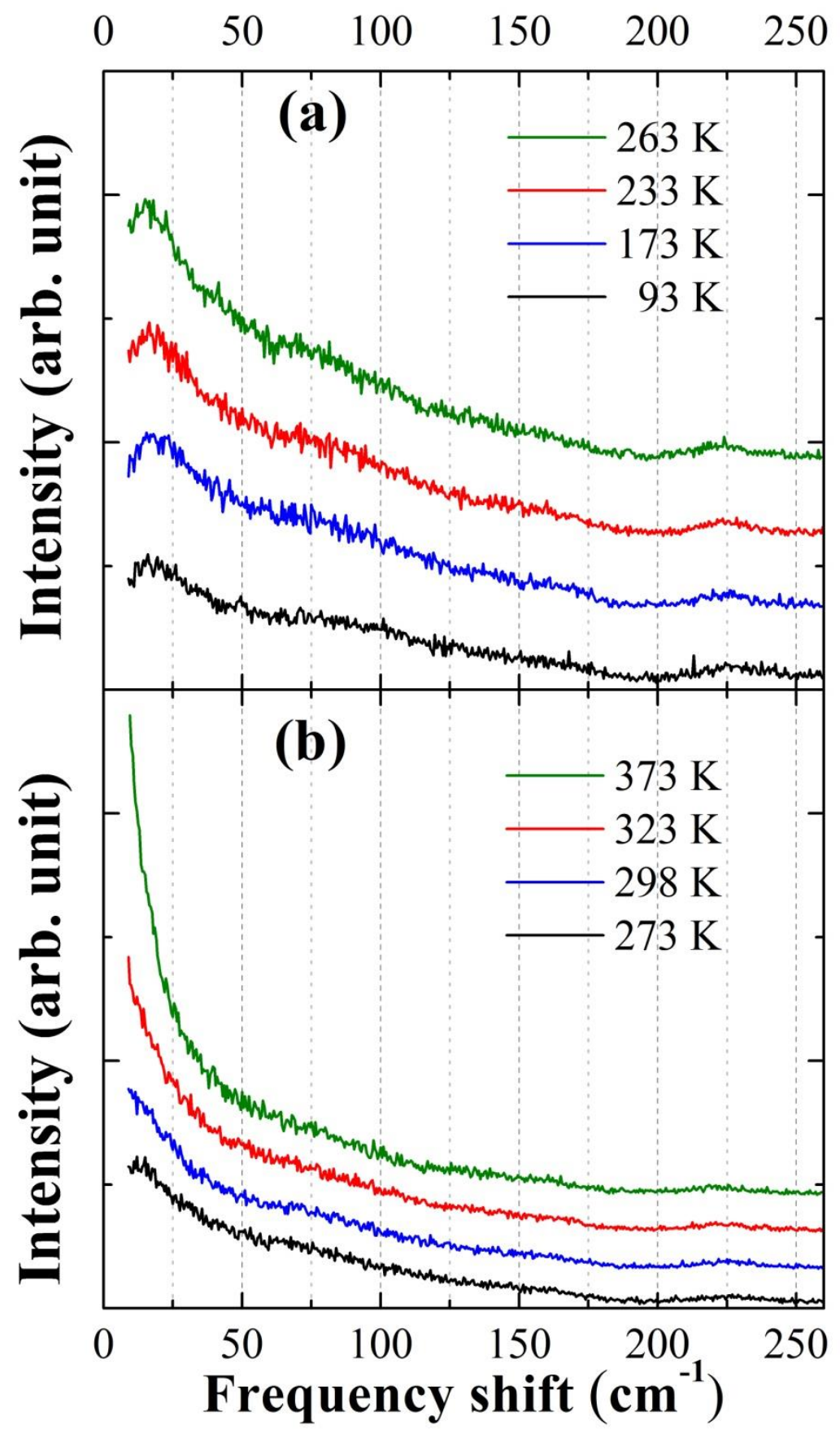


Figure 3

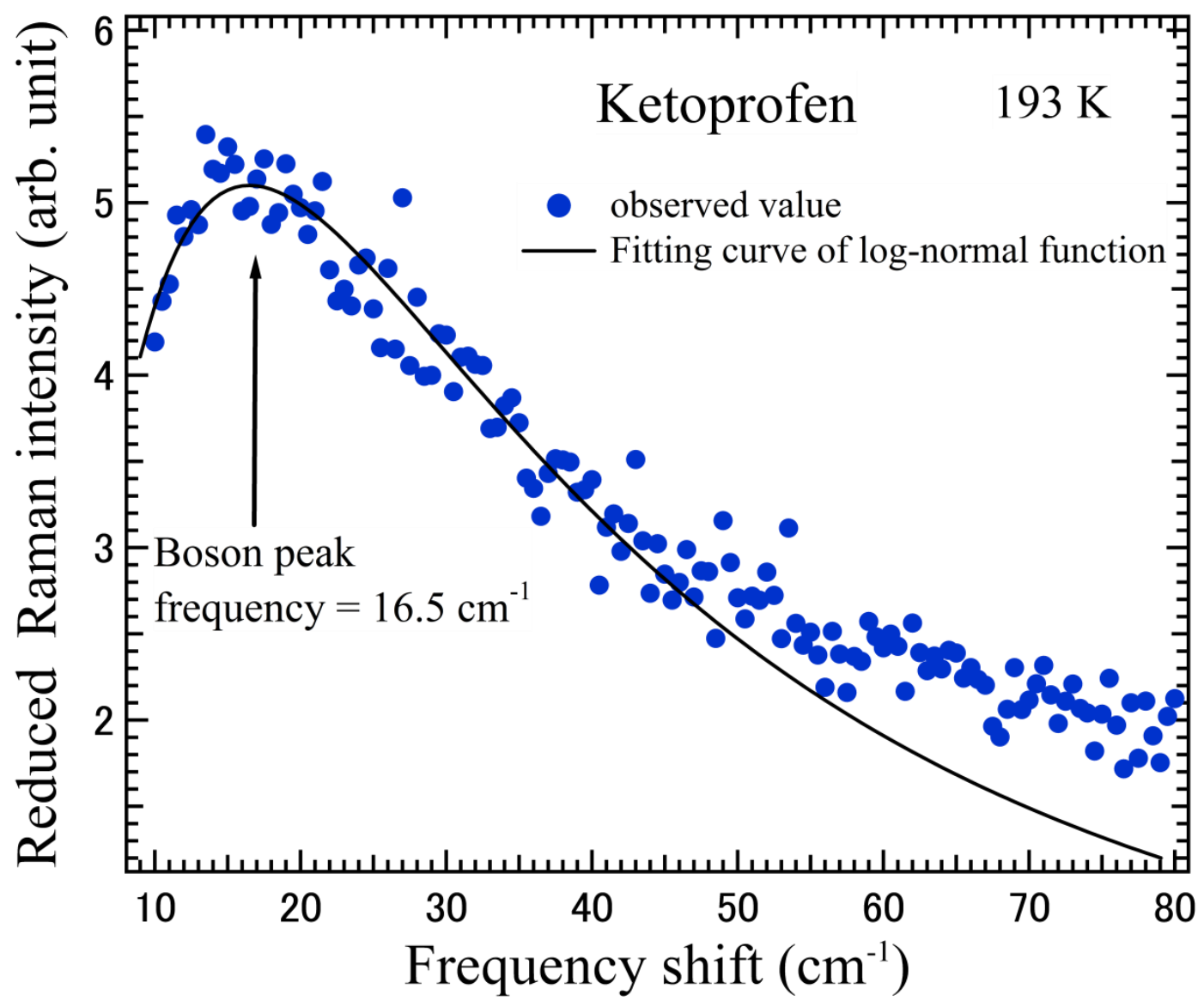


Figure 4

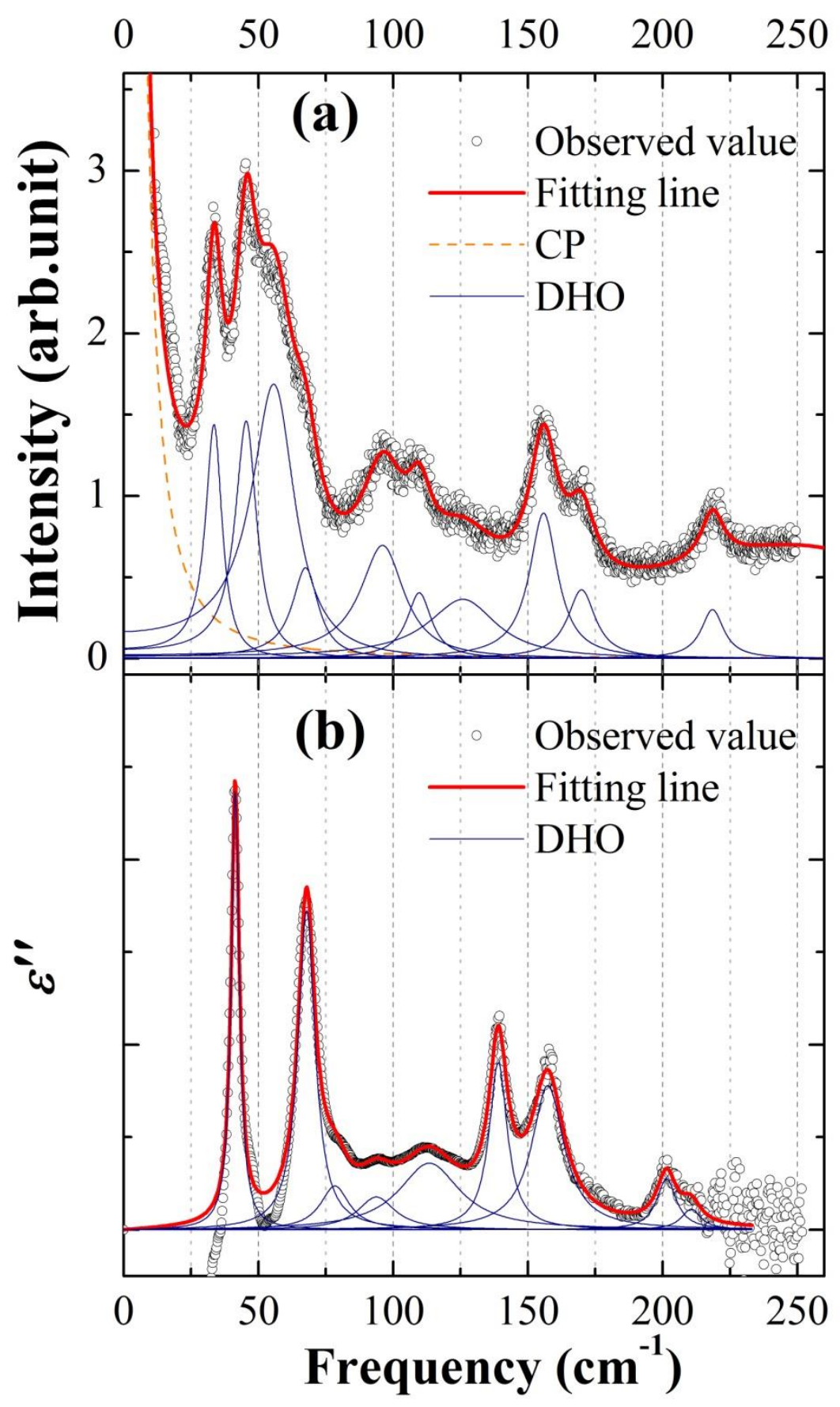




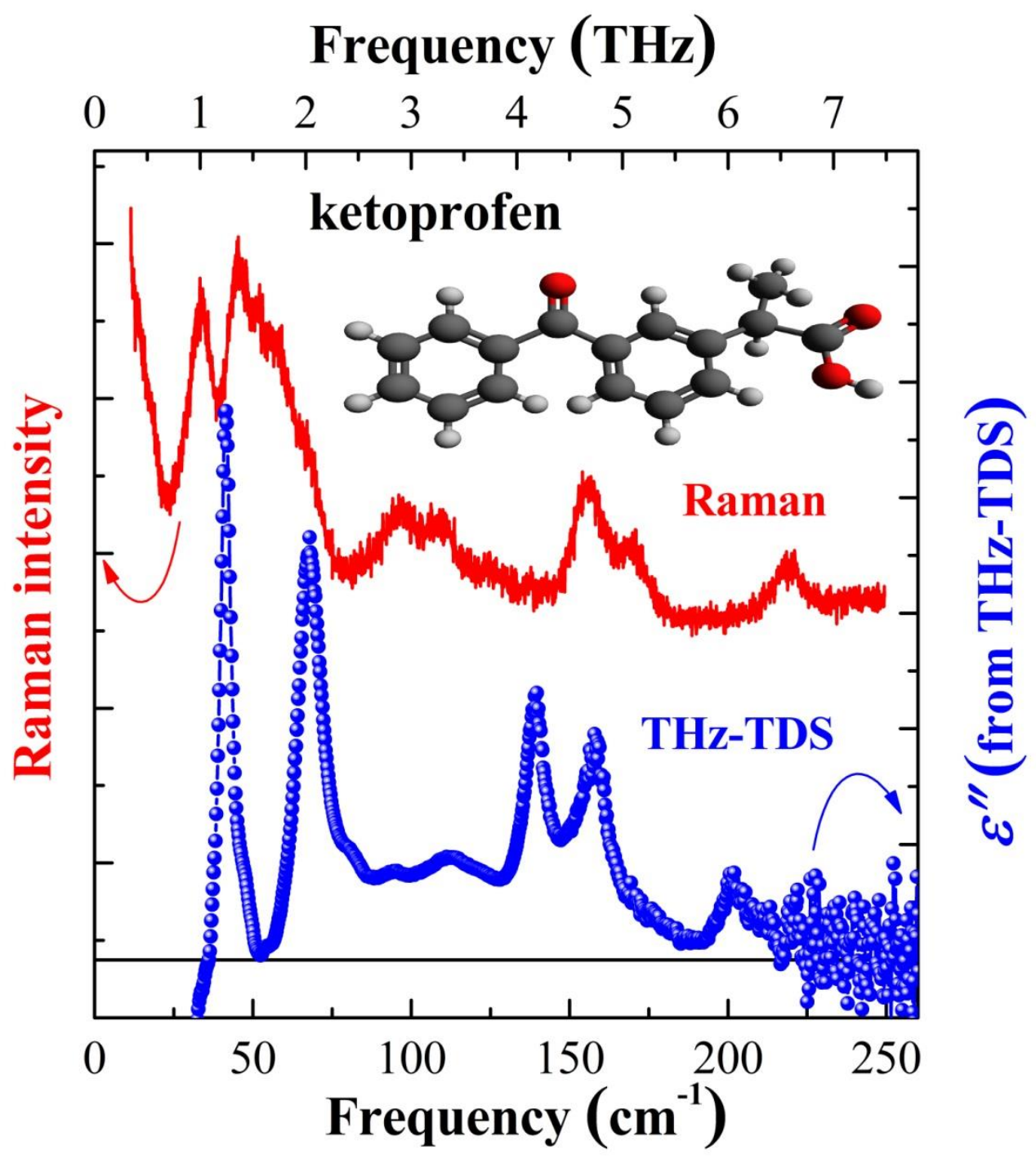




\section{Highlight}

- Vibrational properties of pharmaceutical racemic ketoprofen were investigated.

- Low-frequency Raman scattering spectra clearly showed the boson peak.

- Remarkable change of Raman spectra was observed through a liquid-glass transition.

- The THz-TDS spectra of crystalline state were compared with Raman scattering spectra. 